\title{
Global Mobile Satellite Service Interference Analysis for the AeroMACS
}

Jeffrey D. Wilson and Rafael D. Apaza

Glenn Research Center, Cleveland, Ohio

Ward Hall

ITT Exelis, Fort Wayne, Indiana

Brent Phillips

Federal Aviation Administration, Washington, D.C. 


\section{NASA STI Program . . . in Profile}

Since its founding, NASA has been dedicated to the advancement of aeronautics and space science. The NASA Scientific and Technical Information (STI) program plays a key part in helping NASA maintain this important role.

The NASA STI Program operates under the auspices of the Agency Chief Information Officer. It collects, organizes, provides for archiving, and disseminates NASA's STI. The NASA STI program provides access to the NASA Aeronautics and Space Database and its public interface, the NASA Technical Reports Server, thus providing one of the largest collections of aeronautical and space science STI in the world. Results are published in both non-NASA channels and by NASA in the NASA STI Report Series, which includes the following report types:

- TECHNICAL PUBLICATION. Reports of completed research or a major significant phase of research that present the results of NASA programs and include extensive data or theoretical analysis. Includes compilations of significant scientific and technical data and information deemed to be of continuing reference value. NASA counterpart of peer-reviewed formal professional papers but has less stringent limitations on manuscript length and extent of graphic presentations.

- TECHNICAL MEMORANDUM. Scientific and technical findings that are preliminary or of specialized interest, e.g., quick release reports, working papers, and bibliographies that contain minimal annotation. Does not contain extensive analysis.

- CONTRACTOR REPORT. Scientific and technical findings by NASA-sponsored contractors and grantees.
- CONFERENCE PUBLICATION. Collected papers from scientific and technical conferences, symposia, seminars, or other meetings sponsored or cosponsored by NASA.

- SPECIAL PUBLICATION. Scientific, technical, or historical information from NASA programs, projects, and missions, often concerned with subjects having substantial public interest.

- TECHNICAL TRANSLATION. Englishlanguage translations of foreign scientific and technical material pertinent to NASA's mission.

Specialized services also include creating custom thesauri, building customized databases, organizing and publishing research results.

For more information about the NASA STI program, see the following:

- Access the NASA STI program home page at http://www.sti.nasa.gov

- E-mail your question to help@sti.nasa.gov

- Fax your question to the NASA STI Information Desk at 443-757-5803

- Phone the NASA STI Information Desk at 443-757-5802

- Write to: STI Information Desk NASA Center for AeroSpace Information 7115 Standard Drive Hanover, MD 21076-1320 
NASA/TM-2013-216530

Global Mobile Satellite Service Interference Analysis for the AeroMACS

Jeffrey D. Wilson and Rafael D. Apaza

Glenn Research Center, Cleveland, Ohio

Ward Hall

ITT Exelis, Fort Wayne, Indiana

Brent Phillips

Federal Aviation Administration, Washington, D.C.

Prepared for the

Integrated Communications, Navigation and Surveillance (ICNS) 2013 Conference sponsored by the Institute of Electrical and Electronics Engineers Herndon, Virginia, April 23-25, 2013

National Aeronautics and

Space Administration

Glenn Research Center

Cleveland, Ohio 44135 


\section{Acknowledgments}

The authors gratefully acknowledge the Federal Aviation Administration's support of AeroMACS research at the NASA Glenn Research Center.

Trade names and trademarks are used in this report for identification only. Their usage does not constitute an official endorsement, either expressed or implied, by the National Aeronautics and Space Administration.

Level of Review: This material has been technically reviewed by technical management.

Available from

NASA Center for Aerospace Information 7115 Standard Drive

Hanover, MD 21076-1320
National Technical Information Service 5301 Shawnee Road Alexandria, VA 22312

Available electronically at http://www.sti.nasa.gov 


\title{
Global Mobile Satellite Service Interference Analysis for the AeroMACS
}

\author{
Jeffrey D. Wilson and Rafael D. Apaza \\ National Aeronautics and Space Administration \\ Glenn Research Center \\ Cleveland, Ohio 44135 \\ Ward Hall \\ ITT Exelis \\ Fort Wayne, Indiana 46818 \\ Brent Phillips \\ Federal Aviation Administration \\ Washington, D.C. 20591
}

\begin{abstract}
The AeroMACS (Aeronautical Mobile Airport Communications System), which is based on the IEEE 802.16-2009 mobile wireless standard, is envisioned as the wireless network which will cover all areas of airport surfaces for next generation air transportation. It is expected to be implemented in the 5091 to $5150 \mathrm{MHz}$ frequency band which is also occupied by mobile satellite service uplinks. Thus the AeroMACS must be designed to avoid interference with this incumbent service. Simulations using Visualyse software were performed utilizing a global database of 6207 airports. Variations in base station and subscriber antenna distribution and gain pattern were examined. Based on these simulations, recommendations for global airport base station and subscriber antenna power transmission limitations are provided.
\end{abstract}

\section{Introduction}

The AeroMACS, which is based upon the IEEE 802.162009 mobile wireless standard, is envisioned as a wireless network covering all areas of the airport surface for next generation air transportation (Ref. 1). The system would accommodate all mobile communications requirements including parked and taxiing aircraft, various types of ground vehicles, and personnel as well as connection to fixed assets related to airport safety requirements (such as surveillance and navigation aids, weather sensors, and communications stations).

The AeroMACS is intended to operate in portions of the 5000 to $5150 \mathrm{MHz}$ frequency band, including the 5091 to $5150 \mathrm{MHz}$ span that is authorized on a world-wide basis. It is essential that the AeroMACS service does not interfere with other users in this band. In particular, the allocation of the 5091 to $5150 \mathrm{MHz}$ band to the Earth-to-space fixed-satellite service (FSS), limited to feeder links of non-geostationary satellite systems in the mobile-satellite service (MSS) and utilized by the Globalstar network, will restrict the power levels that will be allowed for the AeroMACS networks. This investigation is focused on helping to establish practical limits on the AeroMACS base station transmissions from airports so that the threshold of interference into Globalstar feeder links is not exceeded. This threshold interference power level for Globalstar at low earth orbit (LEO) has been established at $-157.3 \mathrm{dBW}$ corresponding to a two percent increase of the satellite receiver's noise temperature (Ref. 2).

Previously, the interference power distribution at LEO from the AeroMACS transmitters at the 497 major airports in the contiguous United States was simulated with the Visualyse Professional software (Ref. 3). The results were shown to agree closely with those of a previous study by MITRECAASD (Ref. 4). Both omni-directional and sector antennas were modeled and 5 and $10 \mathrm{MHz}$ channels were considered with a center frequency of $5100 \mathrm{MHz}$.

In Reference 5, the effect of the antenna gain profile on interference power was investigated and the accuracy of the model was improved by including a profile based on measured data. It was assumed that the channel bandwidth is $5 \mathrm{MHz}$ centered at $5100 \mathrm{MHz}$. The effect of the inhomogeneous distribution of airports was examined by comparing with a case having the airports evenly distributed. Also the dependence of the interference power on the number of antenna beams and their directions at the airports was simulated.

In this report, the airport database is extended from 497 up to 6207 airports including additional sites from North America, Europe, and all other continents. Based on the simulations, recommendations for global airport base station antenna power transmission limitations are provided.

\section{Objective}

The Mobile Satellite Service Interference Analysis Ad Hoc Working Group was established by the Radio Technical Commission for Aeronautics (RTCA) SC-223 with the following members: Art Ahrens (Harris), Rafael Apaza 
(NASA), Mike Biggs (FAA), Bruce Eckstein (ITT Exelis), Nikos Fistas (ECTL), Jan Erik Hakegard (SINTE), Ward Hall (ITT Exelis), Brent Phillips (FAA), Armin Schlereth (DFS), Antonio Correas Uson (INDRA), Jeffrey Wilson (NASA), and Natalie Zelkin (ITT Exelis).

The group was assigned this charter: "Define a working method of specifying emissions from all expected AeroMACS future deployments that are compliant with ITU cointerference requirements, to establish two-way link levels with the aircraft to ensure closure of the RF-link without adversely affecting the Globalstar Satellite feeder links. The deliverable would be in the form of Minimum Operational Performance Standard (MOPS) or Standards and Recommended Practices Standard (SARPS) requirements and a technical report delivered to an ICAO technical group via a working paper."

The following describes the analysis method, simulation results, recommendations, and conclusions to support this charter.

\section{Analysis}

The interference modeling was performed with Visualyse Professional Version 7 software from Transfinite Systems Limited (Ref. 6). Details of using this software were provided in (Ref. 7) with the modeling procedure summarized by the following seven steps:

1. Define antenna gain dependence on azimuthal and elevation angles.

2. Locate stations (transmitters and receivers).

3. Specify frequency and bandwidth of carriers.

4. Set up the propagation environment.

5. Set up the links between stations.

6. Define victim and interfering links.

7. Specify desired output, submit run, and analyze results.

Two different antenna gain patterns were used in the simulations. The first is for an $80^{\circ}$ beamwidth sector antenna and is based on the manufacturer's data for the antennas used in the Cleveland airport testbed experiments (Ref. 5). The second is for a $120^{\circ}$ beamwidth sector antenna and is based on the recommendation of the International Telecommunication Union Radiocommunication Sector, ITU-R F.1336-3 (Refs. 8 and 9). The model elevation and azimuthal gain patterns for these two antennas are shown in Figure 1 and Figure 2, respectively.

The locations of the antennas were selected from the Openflights database (Ref. 10) which includes the 6207 global airports shown in Figure 3. Airports that did not have an International Civil Aviation Organization (ICAO) location identifier (a four-character alphanumeric code) were assumed to have minimal traffic and were not included. Transmission is centered at $5100 \mathrm{MHz}$ with a $5 \mathrm{MHz}$ bandwidth. The propagation model utilized basic transmission loss in free space, based on ITU-R Rec. P.525. In Reference 11, 19 scenarios with variations in antenna distribution, airport size, antenna beamwidth, and antenna tilt were simulated. In this report, we will look at only the two most realistic scenarios which are designated as Scenarios A and B and are described below. The maximum simulated cumulative interference power at low earth orbit (hot spot) for these runs was used to establish transmitter power limits.

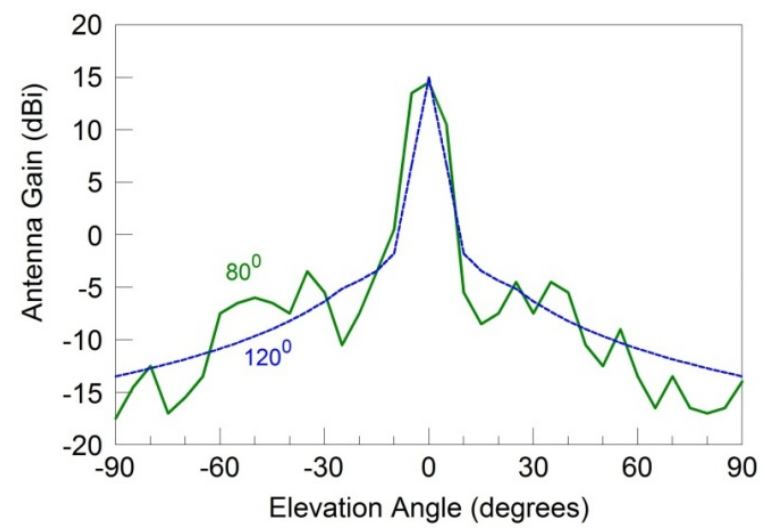

Figure 1.-Modeled Gain Versus Elevation Angle for AeroMACS Antennas

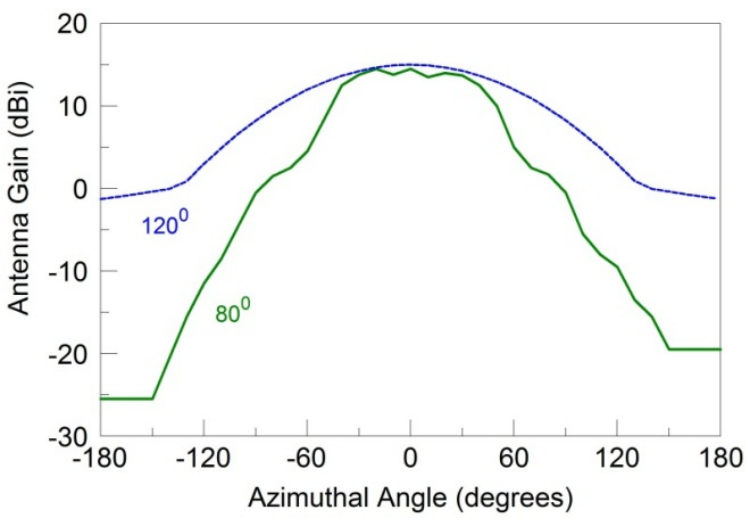

Figure 2.-Modeled Gain Versus Azimuthal Angle for AeroMACS Antennas

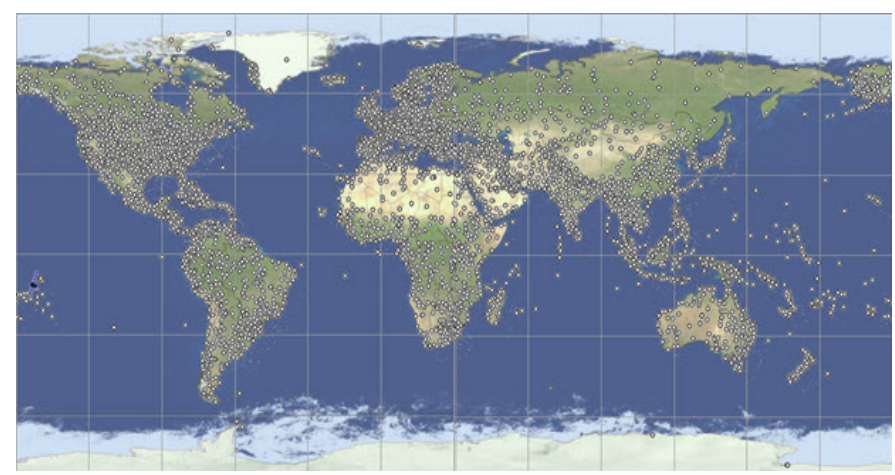

Figure 3.-Locations of 6207 Airports in the Openflights Database 
In each scenario, five different runs were generated with different random antenna directions. The airport sizes were divided into large, medium, and small categories. In the United States, the 35 Operational Evolution Partnership (OEP 35) airports (Ref. 12) are assigned as large airports and the next 123 with the most 2009 passenger boardings from the FAA's Commercial Service Airports CY09 Passenger Boardings list (Ref. 13) are assigned as medium airports. The 50 largest European airports as listed by Wikipedia (Ref. 14) are assigned as large airports and the next 50 on the list are assigned as medium airports. The remaining 5949 airports in the United States, Europe and rest of world from the Openflights database (Ref. 10) are assigned to be small airports. In the model, we assign three $120^{\circ}$ beamwidth sector antennas per channel to each medium airport. The antenna directions are separated by $120^{\circ}$ creating an almost or pseudo omnidirectional azimuthal gain pattern. We assume that the large airports transmit twice as much power per channel as the medium airports and also in a pseudo omnidirectional pattern. This is modeled by using six $120^{\circ}$ sector antennas with directions separated by $60^{\circ}$. Each small airport is assigned a single $120^{\circ}$ sector antenna pointed in a random direction.

In Scenario A, it is assumed that the large airports will use all eleven channels, medium airports will use six channels, and small airports will use just one channel. Thus five out of 11 medium airport and 10 out of 11 small airport transmitters are turned off to model the results for a single channel. Scenario B is the same as Scenario A except that the small airports are only allowed to transmit half as much power per sector as the medium and large airports.

An analysis of subscriber or mobile station transmission limits was also conducted. This evaluation used a model of the antenna system (Ref. 15) employed for mobile measurements conducted at the Next Generation Communication, Navigation, and Surveillance Test Bed (Ref. 16). The measured elevation and azimuthal gain patterns for this antenna are shown in Figure 4 and Figure 5, respectively. The measured elevation gain in Figure 4 was sampled every $5^{\circ}$ as shown in Figure 6 for the Visualyse model. In approximation of the gain pattern of Figure 5, the model azimuthal gain was assumed to be omnidirectional.

With these model gain patterns, two subscriber scenarios based on airport size were considered. In Scenario C, it was assumed that the ratio of subscriber power among small/medium/large airports was 1:4:8. In Scenario D, it was assumed that the ratio was 1:8:16.

\section{Results}

The simulations indicated that the 'hot spot' is most sensitive to the power transmitted from the European airports (Ref. 11). This is because their geographic density is higher than in North America and the other regions. North American airports still have a significant impact and the rest of the world has only a small impact. Figure 7 shows a typical resulting cumulative interference power pattern at low earth orbit. Simulations also showed that the beamwidth does not have a significant effect on the allowable transmitted power.

\section{Radiation Pattern}

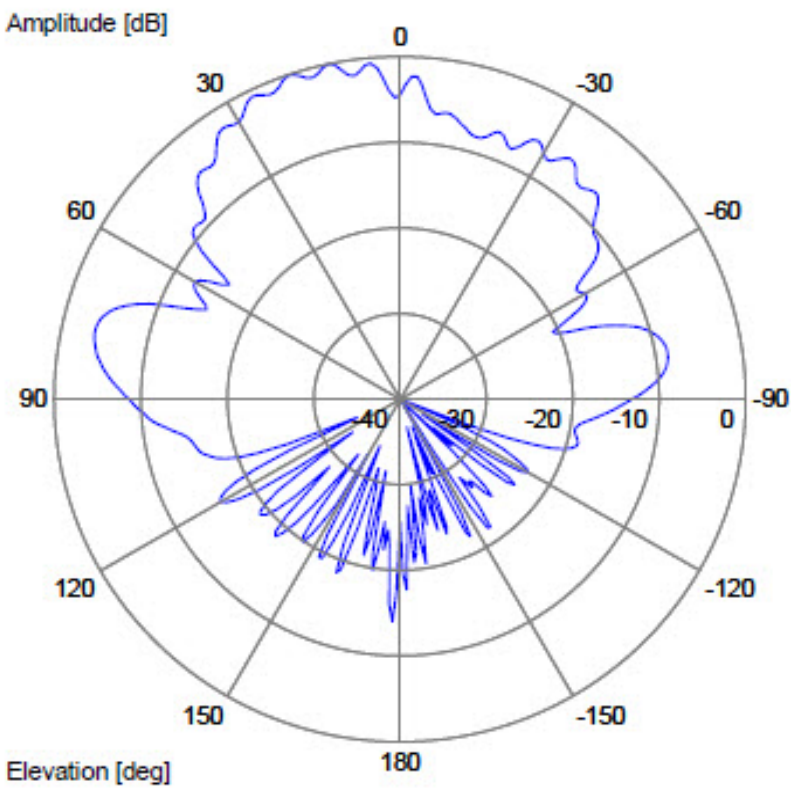

Figure 4.-Measured Gain versus Elevation Angle for Subscriber Antenna (Ref. 15)

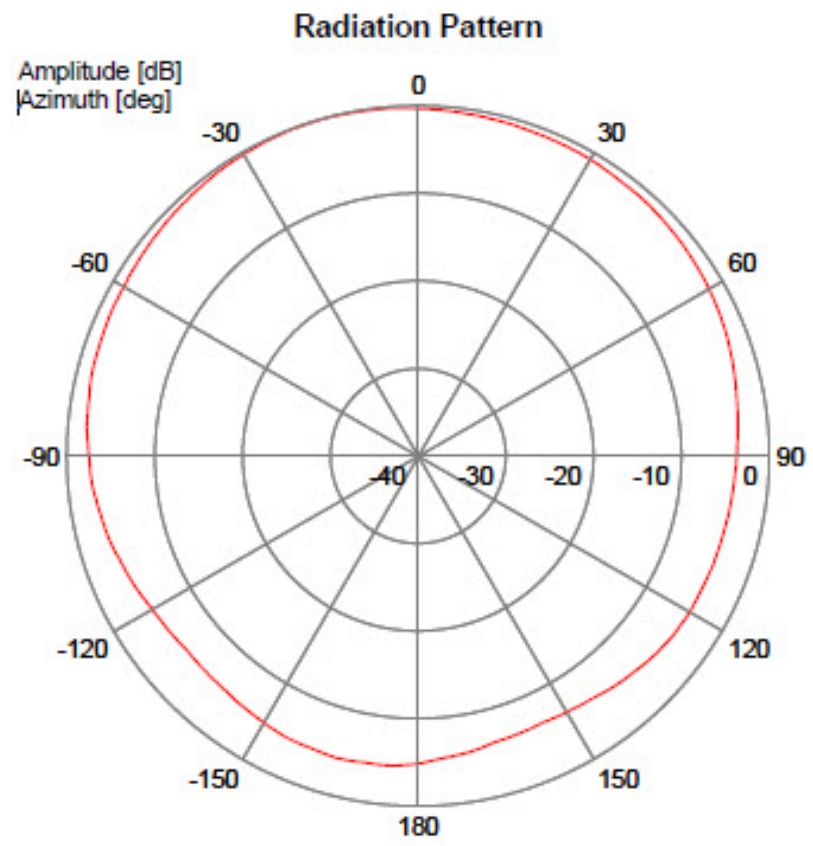

Figure 5.-Measured Gain versus Azimuthal Angle for Subscriber Antenna (Ref. 15) 


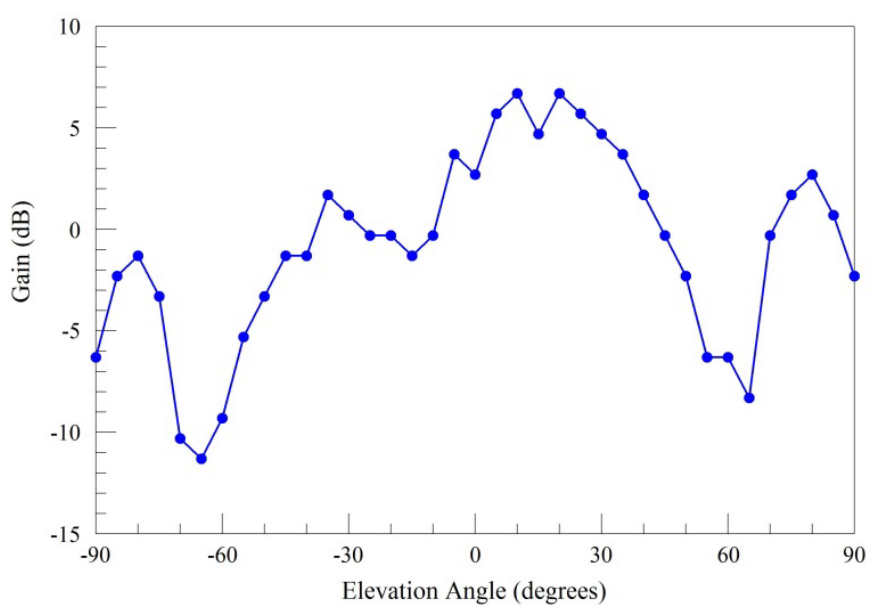

Figure 6.-Model Gain versus Elevation Angle for Subscriber Antenna (Ref. 15)

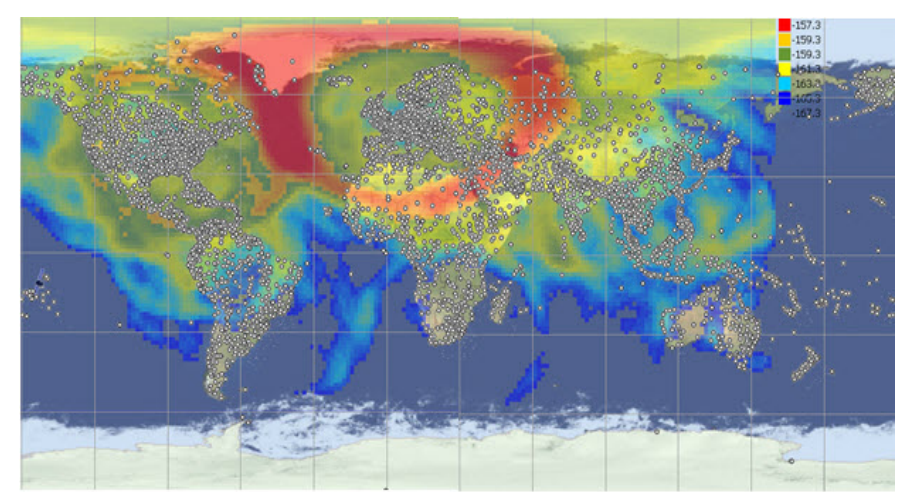

Figure 7.-Typical Cumulative Interference Power Pattern at Low Earth Orbit

Five runs with different random antenna directions were performed for each scenario. The average allowable transmitted power per airport per channel for the scenarios is shown in Figure 8. For Scenario A, large airports could transmit $1711 \mathrm{~mW}$ on each of the eleven channels, medium airports could transmit $855 \mathrm{~mW}$ on each of six channels, and small airports could transmit $285 \mathrm{~mW}$ on one channel. For Scenario B, large airports could transmit $1858 \mathrm{~mW}$ on each of the eleven channels, medium airports could transmit $929 \mathrm{~mW}$ on each of six channels, and small airports could transmit $154 \mathrm{~mW}$ on one channel. Scenario A allows 83 percent more power to be transmitted from small airports than Scenario B but reduces the allowed power transmitted from medium and large airports by only 8 percent.

For the subscriber power transmission simulations, only one run was needed for each of the two scenarios because the model antenna gain pattern is omnidirectional. Figure 9 shows the simulation results of subscriber or mobile station transmission limits per channel for Scenarios C and D where it was assumed that the ratios of subscriber power among small:medium:large airports was $1: 4: 8$ and 1:8:16,

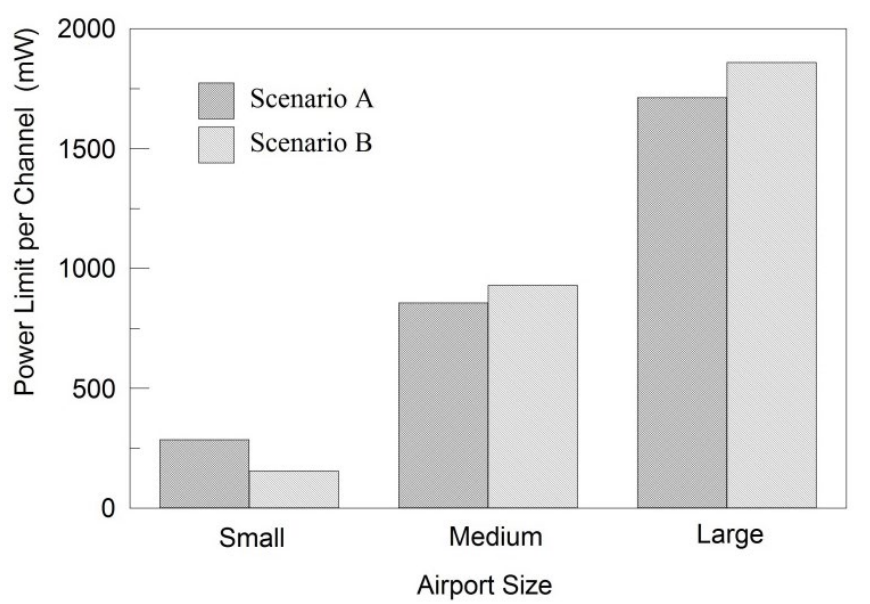

Figure 8.-AeroMACS Base Station Transmission Power Limits

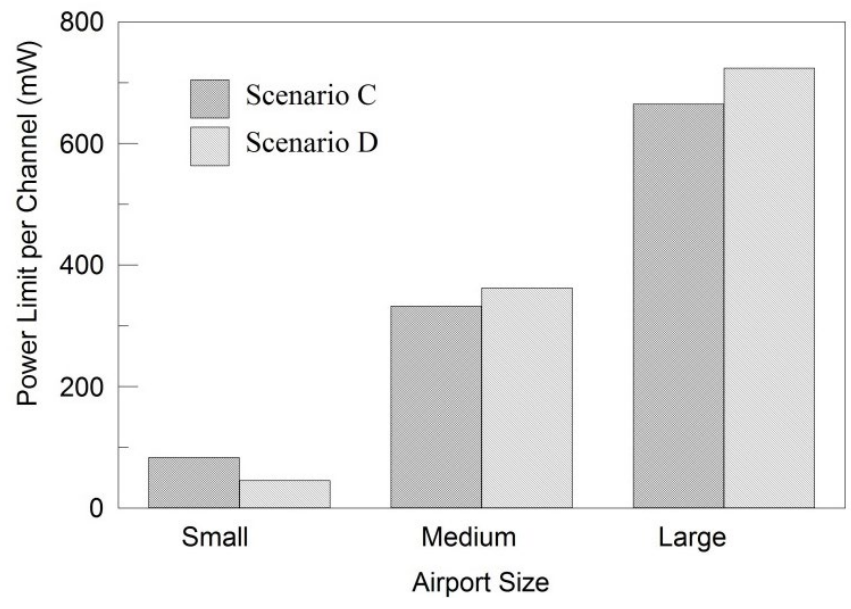

Figure 9.-Subscriber Transmission Power Limits

respectively. For Scenario C, large airports could transmit $664 \mathrm{~mW}$ on each of the eleven channels, medium airports could transmit $332 \mathrm{~mW}$ on each of six channels, and small airports could transmit $83 \mathrm{~mW}$ on one channel. For Scenario $\mathrm{B}$, large airports could transmit $724 \mathrm{~mW}$ on each of the eleven channels, medium airports could transmit $362 \mathrm{~mW}$ on each of six channels, and small airports could transmit $45 \mathrm{~mW}$ on one channel. The power transmission limits for both scenarios is significantly stricter than for the base stations because the subscriber antenna gain is higher at larger elevation angles. Scenario C allows 84 percent more power to be transmitted from small airports than Scenario D but reduces the allowed power transmitted from medium and large airports by only 8 percent.

The scenario in which an equal amount of power was transmitted at each airport regardless of size class was also modeled. For this case the transmission power limit is $237 \mathrm{~mW}$ per channel per airport. It is important to note that the AeroMACS base station and subscriber transmitters cycle so that they are not operating at the same time. 


\section{Recommendations}

According to our classification, the vast majority of global airports are in the small category. Scenarios A and C respectively for the AeroMACS base stations and subscribers allow more than 80 percent more power to be transmitted from small airports than for Scenarios B and D. The allowable transmitted power for medium and large airports is only slightly decreased. Thus we recommend these scenarios as a basis for establishing the power limits which are summarized in Table 1.

TABLE 1.-RECOMMENDED POWER TRANSMISSION LIMITS

\begin{tabular}{|l|c|c|c|}
\hline $\begin{array}{c}\text { Airport } \\
\text { category }\end{array}$ & Channels & $\begin{array}{c}\text { Base station power } \\
\text { per channel, } \\
\mathrm{mW}\end{array}$ & $\begin{array}{c}\text { Subscriber power } \\
\text { per channel, } \\
\mathrm{mW}\end{array}$ \\
\hline Small & 1 & 275 & 80 \\
\hline Medium & 6 & 825 & 330 \\
\hline Large & 11 & 1650 & 660 \\
\hline
\end{tabular}

Power limits are often expressed in terms of the effective isotropic radiated power (EiRP). This is defined as the transmitter power at the antenna input plus the antenna gain.
To determine the EiRP limit as a function of elevation angle, we use a mask based on the elevation pattern of ITU-R F1336-2, "Reference Radiation Patterns of Omnidirectional, Sectoral, and Other Antennas in Point to Multipoint Systems for Use in Sharing Studies in the Frequency Range From $1 \mathrm{GHz}$ to About $70 \mathrm{GHz}$ " (Ref. 8). The pattern and recommended gain mask for the AeroMACS base stations is shown in Figure 10 and Figure 11, for elevation angles between $-100^{\circ}$ to $+100^{\circ}$ and $-900^{\circ}$ to $+900^{\circ}$, respectively.

From the gain mask and the Visualyse simulation results, it is recommended that deployment of the AeroMACS base stations observe the following EiRP limit per channel per sector. (Large, medium, and small airports have respectively 6,3 , and 1 sector antennas per 11, 6, and 1 channels). The total base station EiRP in a single sector (small airports) shall not exceed:

- $\quad 39.4 \mathrm{dBm}$ for elevation angles up to $1.5^{\circ}$

- $39.4 \mathrm{dBm}$ linearly decreasing to $24.4 \mathrm{dBm}$ for elevation angles from $1.5^{\circ}$ to $7.5^{\circ}$

- $24.4 \mathrm{dBm}$ linearly decreasing to $19.4 \mathrm{dBm}$ for elevation angles from $7.5^{\circ}$ to $27.5^{\circ}$

- $\quad 19.4 \mathrm{dBm}$ linearly decreasing to $11.4 \mathrm{dBm}$ for elevation angles from $27.5^{\circ}$ to $90^{\circ}$

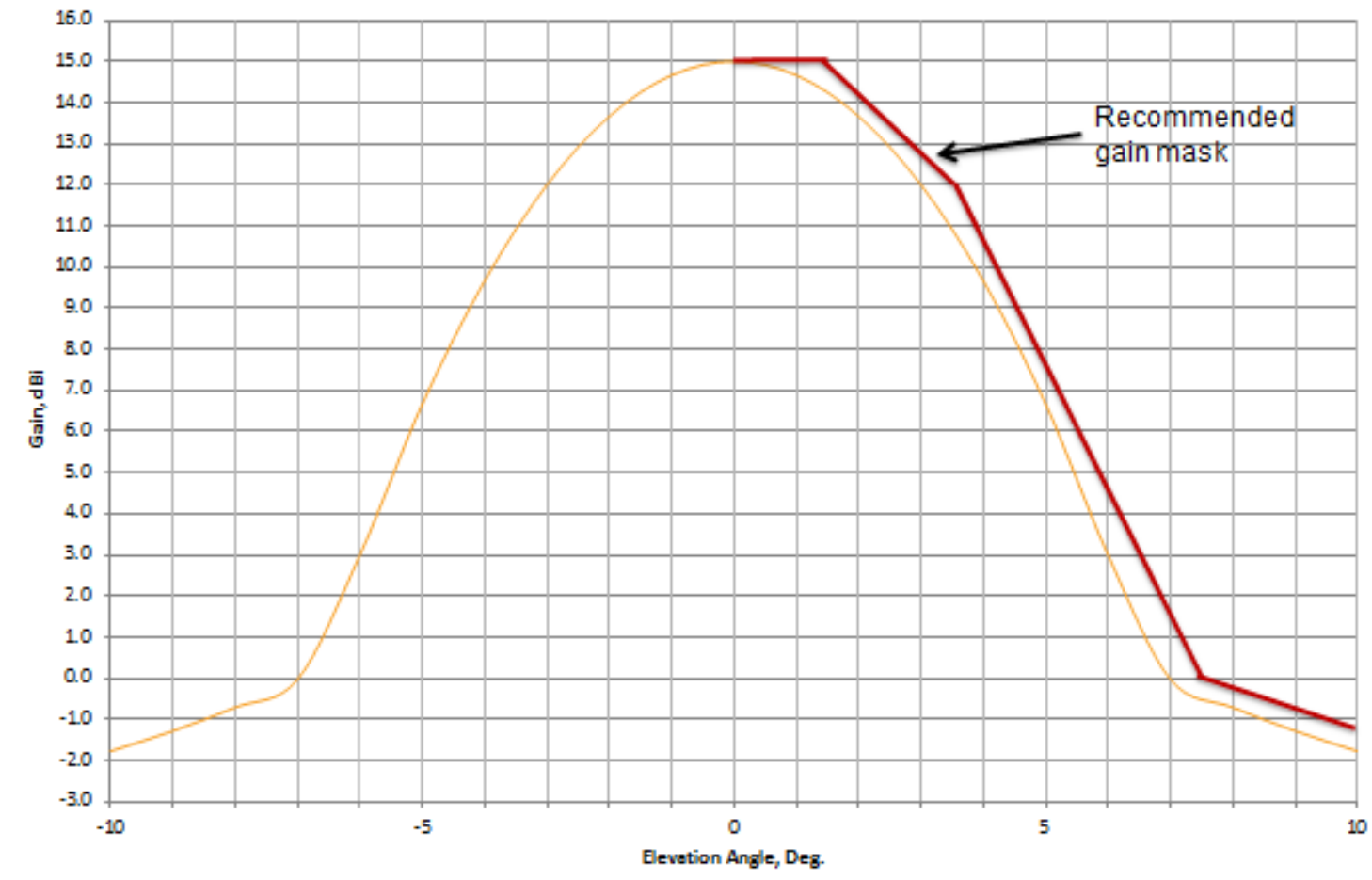

Figure 10.-Elevation Pattern and Mask, $-10^{\circ}$ to $+10^{\circ}$ 


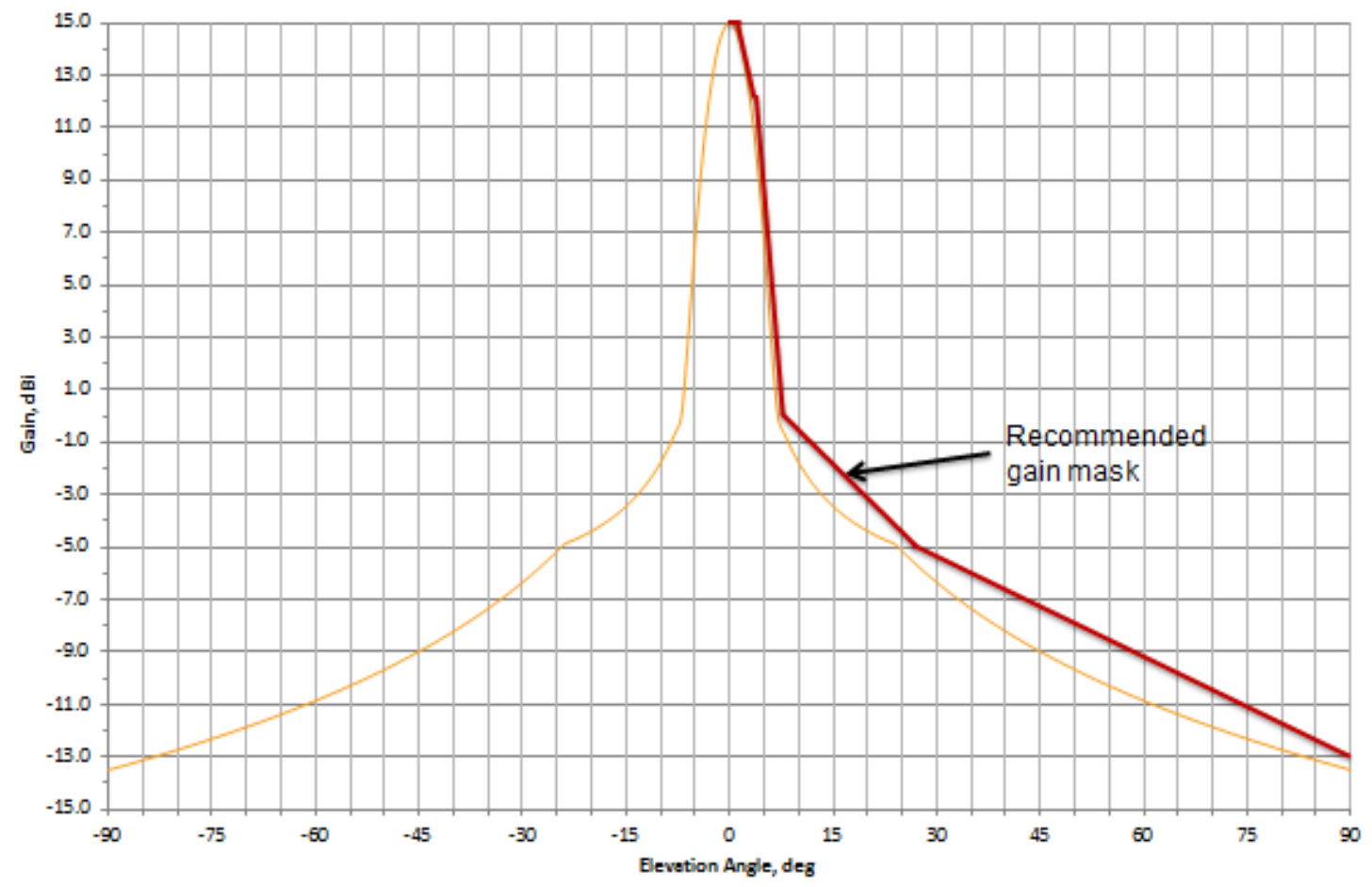

Figure 11.-Elevation Pattern and Mask, $-90^{\circ}$ to $+90^{\circ}$

For medium airports these limits increase by $10 * \log (3)=$ $4.8 \mathrm{dBm}$ and for large airports the limits increase by $10 * \log (6)$ $=6.8 \mathrm{dBm}$. These profiles are shown in Figure 12 .

These limitations include the following assumptions:

(a) EiRP is defined as antenna gain in a specified elevation direction plus the average AeroMACS transmitter power. While the instantaneous peak power from a given transmitter may exceed that level when all of the subcarriers randomly align in phase, when the large number of transmitters assumed in the analysis is taken into account, average power is the appropriate metric.

(b) The breakpoints in the base station EIRP mask are consistent with the elevation pattern of a $+15 \mathrm{dBi}$ peak, $120^{\circ}$ sector antenna as contained in ITU-R F.1336-2.

(c) If a station sector contains multiple transmit antennas on the same frequency (e.g., MIMO), the specified power limit is the sum of the power from each antenna.

(d) No base station antenna down-tilt is applied in these assumptions. Higher sector average transmit power may meet these limitations if antenna pattern down-tilt is used.

(f) The total subscriber EiRP shall not exceed $30 \mathrm{dBm}$. This is based on full occupancy of transmit subcarriers for a $5 \mathrm{MHz}$ bandwidth.

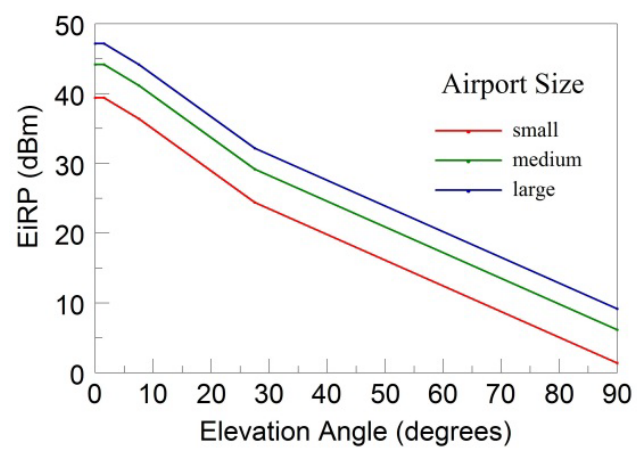

Figure 12.-EiRP Limitations for Small, Medium, and Large Airports

\section{Conclusions}

In order to establish power limits for the AeroMACS base station transmitters to avoid interference with Globalstar uplinks, base stations with sector antenna transmitters were modeled at 6207 airports in the United States, Europe, and the rest of the world with Visualyse Professional software. The maximum simulated cumulative interference power levels at low earth orbit (hot spot) for two scenario options were used to establish transmitter power limits. Transmission power limits were also established for subscribers. 


\section{(a) AeroMACS Base Stations}

In the preferred Scenario A, 85 large airports in the U.S. and Europe can transmit up to about $1650 \mathrm{~mW}$ on each of 11 available channels before the interference threshold is reached. The 173 medium airports in the U.S. and Europe can transmit up to $825 \mathrm{~mW}$ on each of 6 channels and the 5951 small worldwide airports can transmit up to $275 \mathrm{~mW}$ on one channel.

In Scenario B, the allowable power was very significantly reduced for small airports which resulted in a modest increase in allowable power for large and medium airports. In this scenario, the large airports can transmit up to about $1800 \mathrm{~mW}$ on each of 11 available channels, the medium airports can transmit up to $900 \mathrm{~mW}$ on each of 6 channels and the small airports can transmit up to $150 \mathrm{~mW}$ on one channel.

\section{(b) Subscribers}

In the preferred Scenario $\mathrm{C}$, we assume that the ratio of subscriber powers among small/medium/large airports is 1:4:8. Then subscribers at small/medium/large airports can transmit up to $80 / 330 / 660 \mathrm{~mW}$. Alternatively in Scenario D, we assume a 1:8:16 ratio. Then subscribers can transmit up to $45 / 360 / 720 \mathrm{~mW}$ at small/medium/large airports. Finally, if we wish to specify the same limit at all airports regardless of size category, the recommended subscriber transmission limit is $350 \mathrm{~mW}$.

\section{References}

1. Kerczewski, R.J., J.M. Budinger, T.J. Gilbert, 2008, Technology Assessment Results of the Eurocontrol/FAA Future Communications Study, IEEE Aerospace Conference.

2. Gheorghisor, I.L., Y.-S. Hoh, A.E. Leu, 2009, Analysis of ANLE Compatibility with MSS Feeder Links, MITRE-CAASD Report MTR090458.
3. Wilson, J.D., R.J. Kerczewski, 2011, Interference Analysis for an Aeronautical Mobile Airport Communications System, IEEE Aerospace Conference Proceedings, Paper 4.1503, Big Sky, Montana.

4. Hoh, Y.-S., I.L. Gheorghisor, A.E. Leu, 2005, Feasibility Analysis of 5091-5150 MHz Band Sharing by ANLE and MSS Feeder Links, MITRE-CAASD Report MP $05 \mathrm{~W} 0000083$.

5. Wilson, J.D., 2012, Dependence of AeroMACS Interference on Airport Radiation Pattern Characteristics, Integrated Communications, Navigation and Surveillance Conference, Herndon, VA, April 24-26, 2012.

6. http://transfinite.com/content/downloadsvisualyse.html.

7. Wilson, J.D., 2011, Modeling C-Band Co-Channel Interference From AeroMACS Omni-Directional Antennas to Mobile Satellite Service Feeder Uplinks, NASA/TM-2011-216938.

8. ITU-R, 2012, F-1336-3 Reference Radiation Patterns of Omni-directional, Sectoral and Other Antennas in Pointto-Multipoint Systems for Use in Sharing Studies in the Frequency Range from $1 \mathrm{GHz}$ to about $70 \mathrm{GHz}$.

9. J.E. Håkegård, 2011, Compatibility Study between AeroMACS and FSS, Proceedings of 2011 ICNS, Herndon, VA.

10. http://openflights.org/data.html .

11. Wilson, J.D., 2012, Simulating Global AeroMACS Airport Ground Station Antenna Power Transmission Limits to Avoid Interference with Mobile Satellite Service Feeder Uplinks, NASA/TP_-2013-216480.

12. http://aspmhelp.faa.gov/index.php/OEP 35

13. http://www.faa.gov/airports/planning capacity/passenger allcargo stats/passenger/media/cy09 cs enplanements. pdf.

14. http://en.wikipedia.org/wiki/List of the busiest airports in Europe

15. http://www.abcdata.de/abcdataneu/HSPDF/swa 2459 3 $60 \quad 7 \quad 20 \_$v 2.pdf

16. http://nextgentestbed.com/ 



\section{Appendix}

The 497 airports are designated as 'very large', 'large', 'medium', or 'small' according to the number of passenger boardings in calendar year 2009 (Ref. 5):

(a) 10 very large airports with 2009 boardings $>18$ million, modeled with four beams: ATL, ORD, LAX, DFW, DEN, JFK, LAS, IAH, PHX, SFO

(b) 18 large airports with 18 million $>2009$ boardings $>8$ million, modeled with three beams: CLT, EWR, MCO, MIA,
MSP, SEA, DTW, PHL, BOS, IAD, LGA, BWI, FLL, SLC, DCA, SAN, TPA, MDW

(d) 33 medium airports with 8 million $>2009$ boardings $>$ 1.75 million, modeled with two beams: PDX, STL, CVG, MEM, MCI, CLE, OAK, SMF, RDU, BNA, SNA, SJC, HOU, AUS, PIT, MSY, MKE, SAT, IND, DAL, RSW, CMH, PBI, ABQ, JAX, BUF, BDL, ONT, BUR, PVD, OMA, RNO, TUS

(e) 436 small airports with 2009 boardings $<1.75$ million, modeled with one beam: remaining towered airports in contiguous United States. 


